\title{
АНАЛИЗ КОГНИТИВНОЙ МОДЕЛИ ПОЛЕЗНОСТИ ГОСУДАРСТВЕННОГО УПРАВЛЕНИЯ НЕДВИЖИМЫМ ИМУЩЕСТВОМ ОРГАНИЗАЦИЙ
}

\author{
(C) 2018 Камынина Надежда Ростиславовна \\ кандидат технических наук \\ доцент кафедры земельного права и государственной регистрации недвижимости \\ Московский государственный университет геодезии и картографии \\ 105064, г. Москва, Гороховский пер., 4 \\ E-mail: kamyninan@gmail.com
}

В работе представлена постановка когнитивной модели полезности госуправления недвижимым имуществом организаций (НИО) и результаты экспертного определения факторов, проведен статический анализ когнитивной модели полезности госуправления НИО по выявлению наиболее важных факторов полезности с целью повышения качества госуправления НИО страны в целом и формирования стратегии качества госуправления НИО.

Ключевые слова: когнитивный подход, когнитивная модель, полезность госуправления НИО, статический анализ.

Анализ когнитивных карт позволяет получить знания о составе и структуре взаимовлияния составляющих элементов исследуемой управляемой системы [1-8]. В работе [4] выполнена постановка когнитивной карты, однако применение на практике инструментов математического анализа когнитивных карт в сфере управления недвижимостью не выявлено.

Когнитивный подход при реализации практических задач качества госуправления НИО описывает многофакторные управленческие ситуации, что позволит понять их специфику и использовать полученные знания для разработки стратегии качества.

В работах [1-8] показан опыт применения когнитивных карт для моделирования динамики слабоструктурированных ситуаций в экономических системах для решения управленческих проблем, где авторами сформирован и апробирован алгоритм реализации когнитивного подхода, который определяет следующие этапы.

1. Разработка когнитивной модели включает следующие операции: 1.1) постановку целей; 1.2) определение управляющих воздействий; 1.3) проведение факторного анализа; 1.4) экспертное определение перечня наиболее важных факторов; 1.5) определение причинно-следственных связей; 1.6) экспертное определение силы влияния (веса); 1.7) формализацию когнитивной карты.

2. Статический анализ когнитивной карты.
Для реализации методики когнитивного моделирования исследуем полезность госуправления НИО. На первом этапе выявления факторов, влияющих на полезность госуправления НИО, сформировался предварительный перечень SWOT-факторов на основе публичных материалов, опубликованных на официальных сайтах Росреестра, Росимущества, Росстата, Минэкономразвития и т.п. Далее посредством интервьюирования экспертов, обработки результатов опроса с применением вычислительных инструментов формировался свод значимых факторов, связи взаимовлияния и их сила (вес) влияния фактора на фактор в интервале [-1;1], соответствующих лингвистической шкале «сильно-слабо» [1]. Вес определялся в соответствии с установленными правилами оценки межфакторного влияния. Предварительное исследование факторов, описывающих данную ситуацию, позволило сформировать перечень значимых показателей:

1. Х1 Полезность госуправления НИО комплексная (т.е. включает социально - экономическую, правовую, организационную).

2. Х2 Экономический рост.

3. Х3 Финансовый кризис.

4. Х4 Уровень законодательства и общего госуправления.

5. Х5 Санкции.

6. Х6 Уровень процессов в субъектах управления НИО.

7. Х7 Уровень ресурсов для субъектов управления НИО.

8. Х8 Уровень персонала в субъектах НИО. 
Используя описанную методику в [2,3,5], на основе сгенерированной матрицы транзитивного замыкания (табл. 1) сформируем орграф (когнитивную карту) полезности госуправления НИО (рис. 1).

При анализе для изучения статических характеристик взвешенного ориентированного графа используются методы теории графов [11]. В этом случае важной характеристикой выступают циклы обратной связи, существующие на графе. Знак цикла можно определить путем умножения знаков дуг, принадлежащих данному циклу. Положительный цикл усиливает любое начальное изменение фактора, отрицательные циклы противодействуют любым начальным изменениям фактора. В данной модели нет ни отрицательных, ни положительных циклов. Следовательно, можно сделать вывод, что система структурно устойчива.

Ещё одним элементом статического анализа графа когнитивной карты является показатель плотности связей графа. Плотность $d$ для оргра- фа определяется следующим образом:

$$
d=\frac{m}{n(n-1)}
$$

где $m$ - количество дуг в графе когнитивной карты, $n$ - количество факторов в графе. Плотность определяет сложность разработанной модели. Типичные значения плотности находятся в интервале $[0,05,0,3]$. Для данной модели плотность равна 0,23 , что является достаточно высоким показателем [10].

Для когнитивной карты при проведении статического анализа определяется такой параметр как мощность фактора, который даёт оценку важности данного фактора в ее структуре. Важность узла $i\left(i m p_{i}\right)$ оценивается как суммарное количество входящих и исходящих дуг узла.

$$
\operatorname{imp}_{i}=\text { in }_{i}+\text { out }_{i}
$$

где $i n_{i}$, out $t_{i}-$ количество входящих и исходящих дуг узла соответственно.

Таблица 1. Матрица смежности графа

\begin{tabular}{|c|c|c|c|c|c|c|c|c|}
\hline & $\mathrm{X} 1$ & $\mathrm{X} 2$ & $\mathrm{X} 3$ & $\mathrm{X} 4$ & $\mathrm{X} 5$ & $\mathrm{X} 6$ & $\mathrm{X} 7$ & $\mathrm{X} 8$ \\
\hline $\mathrm{X} 1$ & 0 & 0 & 0 & 0 & 0 & 0 & 0 & 0 \\
\hline $\mathrm{X} 2$ & 0,8 & 0 & 0 & 0 & 0 & 0 & 0 & 0 \\
\hline $\mathrm{X} 3$ & $-0,3$ & $-0,5$ & 0 & 0 & 0 & 0 & 0 & 0 \\
\hline $\mathrm{X} 4$ & $-0,2$ & 0,3 & 0 & 0 & 0 & 0 & 0 & 0 \\
\hline $\mathrm{X} 5$ & $-0,1$ & $-0,1$ & 0,3 & 0 & 0 & 0 & 0 & 0 \\
\hline $\mathrm{X} 6$ & 0,8 & 0 & 0 & 0 & 0 & 0 & 0 & 0 \\
\hline $\mathrm{X} 7$ & 0,8 & 0 & 0 & 0 & 0 & 0,5 & 0 & 0 \\
\hline $\mathrm{X} 8$ & 0,9 & 0 & 0 & 0 & 0 & 0,8 & 0 & 0 \\
\hline
\end{tabular}

Источник. Составлено автором.

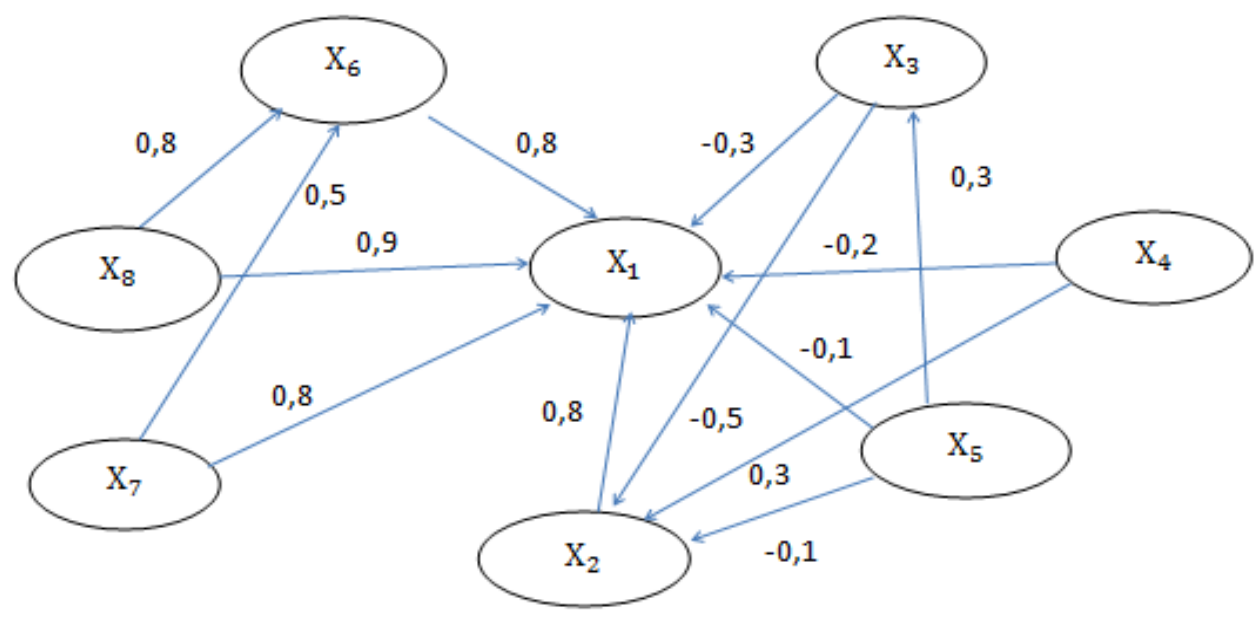

Puc. 1. Когнитивная карта полезности госуправления (оргаф) 
В табл. 2 приводятся данные характеристики для когнитивной карты полезности госуправления НИО. Из таблицы следует, что наиболее важными факторами являются факторы $\mathrm{X}_{1}$ и $\mathrm{X}_{2}$ («Полезность НИО», «Экономический рост»).

Задача прогнозирования развития ситуации решается с помощью матрицы $Q$ и определяется как сумма ряда $E+W+W^{2}+\ldots+W^{t}$.

$$
Q=E+W+W^{2}+\ldots+W^{n} \cong(E-W)^{-1},
$$

где $W$ - матрица смежности графа, $n-$ порядок матрицы $W$, т.е. количество факторов в когнитивной карте. Матрица смежности графа представлена на рисунке 2. В таблице 3 представлена матрица $Q$.

$$
W=\left[\begin{array}{cccccc}
0 & 0 & 0 & 0 & 0 & 00 \\
0,8 & 0 & 0 & 0 & 0 & 00 \\
-0,3 & -0,5 & 0 & 0 & 0 & 00 \\
5 & -0,2 & 0,3 & 0 & 00000 & \\
& -0,1 & -0,10,300000 & \\
0,8 & 0 & 0 & 00000 & \\
& 0,800000,500 & \\
0,900000,800
\end{array}\right.
$$

Puc. 2 - Матрица смежности графа

Для простого импульсного процесса с начальным фактором значение фактора можно вычислить по формуле [11]:

$$
\begin{array}{r}
x_{j}(t)=x_{j}(0)+\{\text { элемент } i, j \text { в матрице } \\
\left.E+W+W^{2}+\ldots+W^{t}\right\}
\end{array}
$$

Например, для простого импульсного процесса с начальной вершиной Х6 (фактор «Уровень процессов в субъектах управления НИО»), при условии нулевых начальных условий $X_{i}(0)=$ $0_{i}=1, \ldots, 8$, фактор X1 («Полезность НИО») увеличится до 0,8 .

Из таблицы 3 также видно, что наибольшее значение фактора X1 может быть достигнуто одновременным воздействием на факторы X2, X4, X6, X7, X8. Данный вывод подтверждается моделированием системы в пространстве состояний с помощью разностных уравнений первого порядка:

$$
\mathbf{x}(t+1)=\boldsymbol{W} \mathbf{x}(t)+\boldsymbol{B} \mathbf{u}(t)
$$

где $\mathbf{x}-$ вектор состояния системы, $\mathbf{u}-$ вектор управления, $\boldsymbol{W}$ - матрица смежности графа, $\boldsymbol{B}-$ матрица весовых коэффициентов управляющих воздействий.

На рисунке 3 представлена зависимость фактора X1 при начальном векторе управления $\mathbf{u}(0)=(0,1,0,1,0,1,1,1)$ и нулевых начальных условиях.

Из рисунка 3 видно, что максимальное зна-

Таблица 2. Мощность факторов модели

\begin{tabular}{|c|c|c|c|c|c|c|c|c|}
\hline & $\mathrm{X}_{1}$ & $\mathrm{X}_{2}$ & $\mathrm{X}_{3}$ & $\mathrm{X}_{4}$ & $\mathrm{X}_{5}$ & $\mathrm{X}_{6}$ & $\mathrm{X}_{7}$ & $\mathrm{X}_{8}$ \\
\hline in $_{i}$ & 7 & 3 & 1 & 0 & 0 & 2 & 0 & 0 \\
\hline out $_{i}$ & 0 & 1 & 2 & 2 & 3 & 1 & 2 & 2 \\
\hline imp $_{i}$ & 7 & 4 & 3 & 2 & 3 & 3 & 2 & 2 \\
\hline
\end{tabular}

Источник. Составлено автором

Таблица 3 - Матрица транзитивного замыкания

\begin{tabular}{|c|c|c|c|c|c|c|c|c|}
\hline & $\mathrm{X} 1$ & $\mathrm{X} 2$ & $\mathrm{X} 3$ & $\mathrm{X} 4$ & $\mathrm{X} 5$ & $\mathrm{X} 6$ & $\mathrm{X} 7$ & $\mathrm{X} 8$ \\
\hline $\mathrm{X} 1$ & 1 & 0 & 0 & 0 & 0 & 0 & 0 & 0 \\
\hline $\mathrm{X} 2$ & 0,8 & 1 & 0 & 0 & 0 & 0 & 0 & 0 \\
\hline $\mathrm{X} 3$ & $-0,7$ & $-0,5$ & 1 & 0 & 0 & 0 & 0 & 0 \\
\hline $\mathrm{X} 4$ & 0,04 & 0,3 & 0 & 1 & 0 & 0 & 0 & 0 \\
\hline $\mathrm{X} 5$ & $-0,39$ & $-0,25$ & 0,3 & 0 & 1 & 0 & 0 & 0 \\
\hline $\mathrm{X} 6$ & 0,8 & 0 & 0 & 0 & 0 & 1 & 0 & 0 \\
\hline $\mathrm{X} 7$ & 1,2 & 0 & 0 & 0 & 0 & 0,5 & 1 & 0 \\
\hline $\mathrm{X} 8$ & 1,54 & 0 & 0 & 0 & 0 & 0,8 & 0 & 1 \\
\hline
\end{tabular}

Источник: составлено автором 


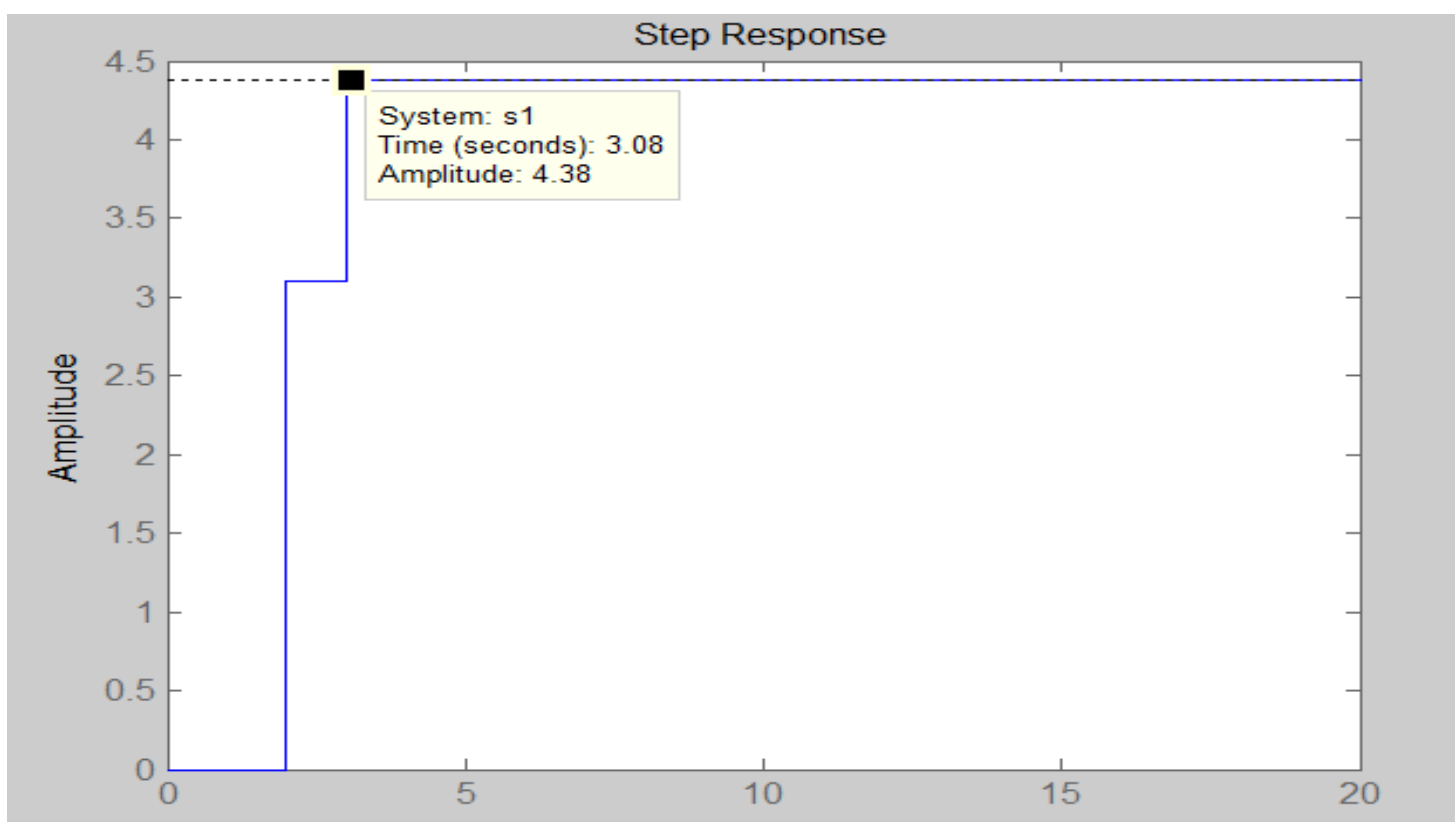

Puc. 3. Значение фактора X1 при выбранном векторе управления

чение целевого фактора достигается через три периода управленческого воздействия.

В целом наибольшее значение полезности НИО может быть достигнуто при одновременном управленческом воздействии на факторы X2 (Экономический рост), X4 (Уровень законодательства и общего госуправления), Х6 (Уровень процессов в субъектах управления НИО), $\mathrm{X} 7$ (Уровень ресурсов для субъектов управления НИО), Х8 (Уровень персонала в субъектах НИО). Вместе с тем, если предполагается стагнация в экономике, то фактор Х2 (Экономический рост) становится недостаточно влиятельным, следовательно, переходим постепенно к тем факторам, которые имеют большую динамику. Такими факторами будут выступать X6 (Уровень процессов в субъектах управления НИО), Х7 (Уровень ресурсов для субъектов управления НИО), Х8 (Уровень персонала в субъектах НИО).

Однако при недостаточности ресурсов параметр X7 (Уровень ресурсов для субъектов управления НИО) теряет значимость для обеспечения роста фактора X1. Соответственно, необходимо оказывать управленческое воздействие на факторы Х8 (Уровень персонала в субъектах НИО) и Х6 (Уровень процессов в субъектах управления НИО).

Таким образом, в ходе исследования сформулирована постановка когнитивной модели, определены факторы полезности госуправления НИО. Статический анализ когнитивной модели показал, что наиболее важными факторами являются «Полезность НИО», «Экономический рост». При этом наибольшее значение фактора «Полезность НИО» может быть достигнуто одновременным воздействием на факторы «Экономический рост», «Уровень законодательства и общего госуправления», «Уровень процессов в субъектах управления НИО», «Уровень ресурсов для субъектов управления НИО», «Уровень персонала в субъектах НИО». Вместе с тем при неблагоприятных экономических условиях факторы Х8 (Уровень персонала в субъектах НИО) и Х6 (Уровень процессов в субъектах управления НИО) становятся ключевыми для управленческого воздействия.

\section{Библиографический список}

1. Абрамова, Н.А., Воронина, Т.А., Федотов А.А. О механизмах формирования оценок значений факторов и весов влияний в когнитивных картах //Труды IX Всероссийской школы-конференции молодых ученых «Управление большими системами», 2012. Т.1. С. 111-114.

2. Авдеева, 3.К., Коврига, С.В. Подход к постановке задач управления на когнитивной модели ситуации для стратегического мониторинга // Управление большими системами: сборник трудов. 2016. Выпуск 59. C. 120-146.

3. Авдеева, 3.К., Коврига, С.В. Формирование стратегии развития социально-экономических объектов на основе когнитивных карт. Saarbrucken: LA PLAMBERT Academic Publishing GmbH\&Co. KG. 2011., 184 c. 
4. Гербеева, Л.Ю. Методология управления собственностью на территории региона: дисс... доктора экономических наук: 08.00.05 / Л.Ю. Гербеева.-Оренбург, 2015.- 377 с.

5. Горелова, Г.В., Захарова, Е.Н., Радченко, С.А. Исследование слабоструктурированных проблем социально-экономических систем. Когнитивный подход. Ростов н/Д. 2006., 332 с.

6. орноушенко, Е. К., Максимов, В.И. Управление ситуацией с использованием структурных свойств ее когнитивной карты / / Тр. Ин-та пробл. упр. им. В.А. Трапезникова РАН. Москва. 2000. Т. ХІ. С. 85-90.

7. ульба, В.В., Кононов, Д.А., Косяченко, С.А., Шубин, А.Н. Методы формирования сценариев развития социально-экономических систем Москва. 2004., 296 с.

8. В.Н. Волкова, Г.В. Горелова, В.Н. Козлов [и др.] Моделирование систем и процессов: учебник для академического бакалавриата / под ред. В.Н. Волковой, В.Н. Козлова. Москва. 2014., 588 с.

9. Робертс, Ф.С. Дискретные математические модели с приложением к социальным, биологическим и экологическим задачам/ Пер. с англ. А.М. Раппопорта, С.И. Травкина под ред. А.И. Теймана. Москва. $1986 ., 496$ с.

10. Hart, J.A. (1977). Cognitive maps of three Latin American policy makers. World Polit 30, 1977.-Pp. 115-140.

11. Roberts, F.S. (1976). Discrete mathematical models with applications to social, biological and environmental problems. Prentice-Hall, Englewood Cliffs, 1976. - 560 p. 